\title{
Learning Shape Variations of Motion Trajectories for Gait Analysis
}

\author{
Maxime Devanne \\ Hazem Wannous and Mohamed Daoudi \\ University of Lille 1, Telecom Lille \\ CRIStAL Laboratory (UMR 9189) \\ Villeneuve d'Ascq, France \\ Email: maxime.devanne@telecom-lille.fr
}

\author{
Stefano Berretti \\ Alberto Del Bimbo and Pietro Pala \\ University of Florence and \\ Media Integration and Communication Center \\ Florence, Italy
}

\begin{abstract}
The analysis of human gait is more and more investigated due to its large panel of potential applications in various domains, like rehabilitation, deficiency diagnosis, surveillance and movement optimization. In addition, the release of depth sensors offers new opportunities to achieve gait analysis in a non-intrusive context. In this paper, we propose a gait analysis method from depth sequences by analyzing separately each step so as to be robust to gait duration and incomplete cycles. We analyze the shape of the motion trajectory as signature of the gait and consider shape variations within a Riemannian manifold to learn step models. During classification, the derivation of each performed step is evaluated in an online manner to qualitatively analyze the gait. Experiments are carried out in the context of abnormal gait detection and person re-identification trough gait recognition. Results demonstrated the potential of the method in both scenarios.
\end{abstract}

\section{INTRODUCTION}

The analysis of human motion through vision-based techniques has been widely investigated in recent years mainly due to its large spectrum of potential applications in various domains, like entertainment, medicine, surveillance and sport [3]. More recently, the release of new depth sensors, like Microsoft Kinect offered new opportunities to analyze human motion, by providing in real-time 3D skeleton data representing the human pose. These data have been successfully used to support fall detection [16], action recognition [8], [24], motion segmentation [7], [25] and person authentication [1], [11].

In particular, a growing attention has targeted the analysis of human gait by health professionals and researchers. It has been shown that human gait is an important indicator of health and well being [12]. Hence, a qualitative evaluation of gait is appreciated and several measures of quality have been investigated [6], [22]. However, the measurement of gait parameters are often based on expensive or intrusive systems, like force plates, markers or wearable sensors. Therefore, such systems can be employed only in laboratories and require deliberate user cooperation. In order to face these constraints, the use of non-intrusive, portable and low-cost depth sensors has been evaluated. Particularly, the accuracy of the estimated skeleton data is evaluated through various measurements, like stride duration and arm angular velocities [10], [23]. Experiments

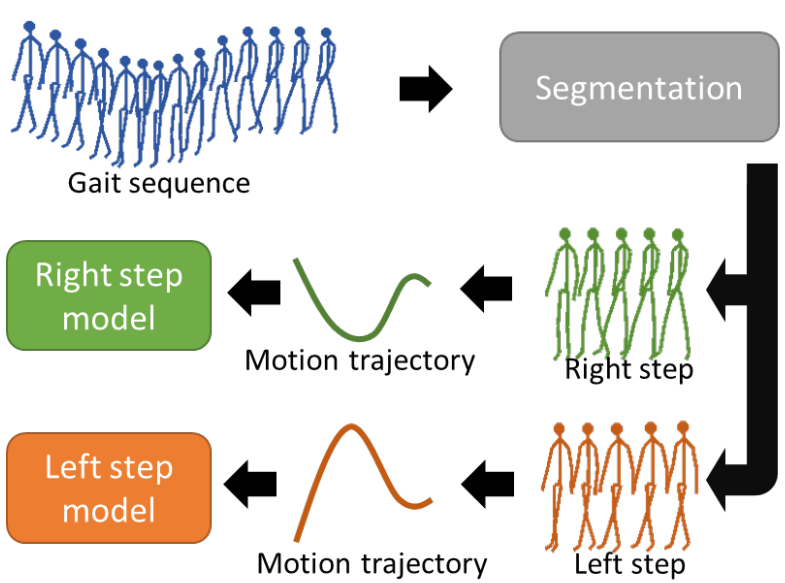

Fig. 1. Overview of our approach. The skeleton gait sequence is first segmented to identify right and left steps. Each step is then represented as a motion trajectory. The shape of trajectories are analyzed in the shape space and shape variations are considered to learn a step model for both steps.

demonstrated that such data are sufficiently robust and accurate for gait analysis in various contexts.

These considerations motivated researchers to analyze the gate of people using data provided depth sensors for the purpose of abnormal gait detection and/or person re-identification. In this paper, we propose an approach for gait analysis based on $3 \mathrm{D}$ data provided by a Kinect sensor. The approach relies on decomposing the observed motion sequence into the left and right steps. The shape of the motion trajectory corresponding to each step is analyzed to define a statistical model capturing the shape of both steps. Finally, these models are used to evaluate, in an online manner, the gait of test subjects for abnormal gait detection and person re-identification. Fig. 1 illustrates the proposed approach.

\section{RELATED WORK}

While the detection of abnormal human behaviors trough visual information has been widely investigated in the last decades [19], only few recent works addressed the problem of gait analysis for abnormal gait detection using depth sensors. Considering that the number of possible abnormality variations 
is often large, learning a robust abnormal gait representation is not convenient. Hence, a common way is to only learn a normal pattern and consider the deviations from this pattern to detect abnormality. The work proposed in [18] uses skeleton data and employs a nonlinear manifold learning technique to reduce the dimensionality and the noise. Then, two statistical models, corresponding to human pose and motion dynamic are learned from the normal gait samples. The evaluation of a test gait sequence is finally achieved by matching the observations to the models in a frame-by-frame manner, thus allowing online processing. The method is applied to detect simulated gait anomalies on subjects climbing a stair. A more recent work [2] applies the joint motion history feature (JMH) to normalized skeleton data to simultaneously capture both the human posture and motion within a short temporal window. Such features are used in a bag-of-word paradigm to describe gait sequences with a set of exemplar features. Comparison between sequences is achieved using Dynamic Time Warping, while classification is obtained by thresholding the similarity measures. However, such sequence-based classification requires the observation of the full sequence before classifying the sequence as normal or abnormal. In addition, the method is not suitable to localize the abnormality within the sequence. Finally, in order to analyze the entire sequence, the work proposed in [17] employs a sliding window technique to explore the evolution of interjoints distances as spatio temporal intrinsic feature.

Person identification through gait analysis, as an interesting and non-intrusive biometric means, has been extensively studied in recent years. Several methods have been proposed for gait recognition by using depth or skeleton data captured by RGB-D sensors. Sivapalan et al. [21] propose the Gait Energy Volume feature averaging the voxel volumes, derived from depth images, over an entire gait cycle. An extended feature is proposed in [4] by combining depth and RGB data to reduce noise and considering only a set of key poses during the gait cycle. Conversely, other methods propose to investigate skeleton data to compute features. Preis et al. [20] employ skeleton data to define thirteen biometric features including subject height, length of several limbs, length of steps and speed. Finally, Chattopadhyay et al. [5] combine front and back views of the gait to compute their features. Due to the poor accuracy of skeleton data of the back view, depth-based features are computed in addition to skeleton features from the front view.

\section{ShAPE ANALYSIS OF CURVES IN $\mathbb{R}^{n}$}

In this Section, we briefly describe the mathematical tool, first proposed in [13], that we employ to analyze the shape of both legs poses and body motion. As described below, legs poses and body motion are characterized by curves in $\mathbb{R}^{3}$ and $\mathbb{R}^{60}$, respectively. Let a curve in $\mathbb{R}^{n}$ be represented as $\beta: I \rightarrow \mathbb{R}^{n}$, with $I=[0,1]$. The shape of $\beta$ is mathematically represented through the square-root-velocity function (SRVF) [13], denoted by:

$$
q(t)=\frac{\dot{\beta}(t)}{\|\dot{\beta}(t)\|},
$$

with $t \in[0,1]$. The initial curve $\beta$ can be retrieved (up to a translation) from its SRVF using:

$$
\beta(t)=\int_{0}^{t}\|q(s)\| q(s) d s .
$$

This is particularly useful to visualize effects of shape transformation on curve in $\mathbb{R}^{n}$. The effectiveness of such specific representation for shape analysis has been proven in different applications, like 2D silhouettes [13] and 3D facial curves [9]. Let us define the space of SRVFs as $\mathcal{C}=\left\{q: I \rightarrow \mathbb{R}^{n},\|q\|=\right.$ 1) $\subset \mathbb{L}^{2}\left(I, \mathbb{R}^{n}\right)$, with $\|$.$\| indicating the \mathbb{L}^{2}$ norm. With the $\mathbb{L}^{2}$ metric on its tangent space, $\mathcal{C}$ becomes a Riemannian manifold. Since elements of $\mathcal{C}$ have a unit $\mathbb{L}^{2}$ norm, $\mathcal{C}$ is a hyper-sphere in the Hilbert space $\mathbb{L}^{2}\left(I, \mathbb{R}^{n}\right)$. Consequently the distance between two elements $q_{1}$ and $q_{2}$ is defined as $d_{\mathcal{C}}\left(q_{1}, q_{2}\right)=\cos ^{-1}\left(\left\langle q_{1}, q_{2}\right\rangle\right.$. Such distance represents the similarity between the shape of two curves in $\mathbb{R}^{n}$. Basically, it quantifies the amount of deformation between two shapes. Another advantage of such framework is that it offers some statistical tools. For example, the mean shape $\mu$ among a set of $n$ sample shapes is computed as the Riemannian center of mass [14]:

$$
\mu=\arg \min \sum_{i=1}^{n} d_{\mathcal{C}}\left(\mu, q_{i}\right)^{2} .
$$

\section{GAIT SEGMENTATION}

Gait can be naturally defined as the succession of a right and left step representing a gait cycle. However, the number of gait cycles may differ from one sequence to another. In addition, a gait cycle can start by either the right and left step. When analyzing gait sequences, taking into consideration these two aspects is relevant. Hence, we propose to segment gait sequences in order to study separately the left and right steps. Our goal is to find cutting points corresponding to transition between one step to the other. For that, we propose to analyze the evolution of the limbs motion along the sequence. We consider that within a time interval corresponding to transition between two steps, the motion of limbs is low. Conversely, within a time interval corresponding to step performance, the motion of limbs is higher. To characterize the limb motion, we propose to analyze the shape variation of the two legs. From skeleton data provided by depth sensors, we use joints corresponding to legs in addition to the hip center to build a 3D curve by connecting these joints, as shown in Fig. 2.

To capture and analyze the shape evolution of such 3D curve, we employ the Riemannian shape analysis described in Sect. III within a sliding window technique. Let $q_{i}, i=1 \ldots N$ be the set of curve shapes corresponding to legs poses within a window of size $N$. To quantify the legs motion during the corresponding interval, we compute the standard deviation $\sigma=\frac{1}{N} \sum_{i=1}^{N} d_{\mathcal{C}}\left(\mu, q_{i}\right)$, where $\mu$ corresponds to the mean shape computed according to Eq. (3). We identify transitions 


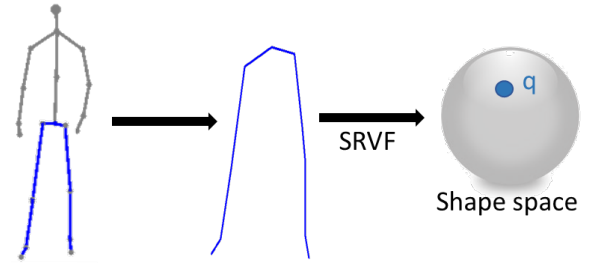

Fig. 2. Shape analysis of leg curve. From the skeleton frame, we consider the leg joints to build a 3D curve. The shape of such 3D curve is analyzed in the shape space.

between two steps by detecting the local minima of the standard deviation. As a result, a gait sequence is now represented as a set of temporal segments corresponding to either right or left steps. Fig. 3 shows the evolution of the standard deviation along a gait sequence. We can observe that temporal segments between two subsequent minima correspond to one step.

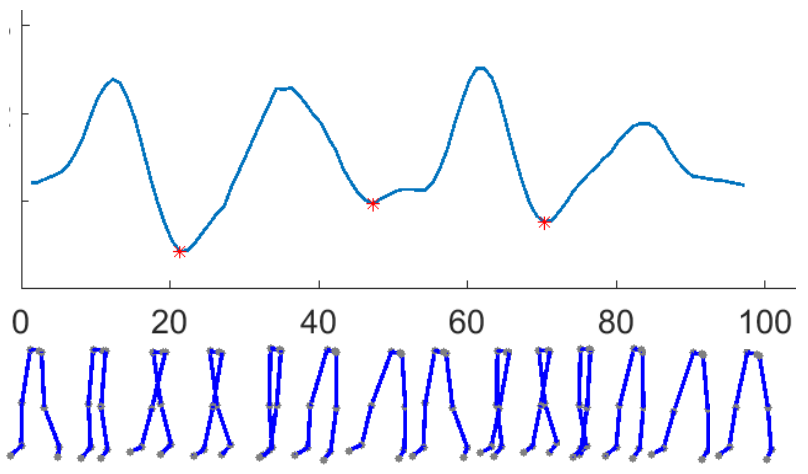

Fig. 3. Standard deviation evolution. By detecting local minima (red), we are able to identify temporal segments corresponding to steps of the gait sequence.

\section{Gait ANALYSis}

Once a gait sequence is segmented into a set of left and right steps, we propose to analyze each step separately. First, we consider the trajectory made by the human body during a step. While most of the works only analyzes the legs motion, here we consider the full body as we believe that arms motion also provide useful information characterizing each step. Let $p_{i}(t)=\left(x_{i}(t), y_{i}(t), z_{i}(t)\right)$ be the 3D position of the joint $i$ at frame $t$. For each frame $t$, we concatenate the $3 \mathrm{D}$ position of each joint to build a vector $v(t)=\left[p_{1}(t), \ldots, p_{J}(t)\right]$, where $J$ is the number of joints of a skeleton. The trajectory $\beta$ made by the skeleton along the steps is obtained by concatenating all the feature vectors of the corresponding time interval $\left[t_{a}, t_{b}\right]$ : $\beta=\left[v\left(t_{a}\right), \ldots, v\left(t_{b}\right)\right]$. Finally, in order to compare such step trajectories lying in a $3 J$-dimensional space, we propose to analyze their shape by using the SRVF described in Sect. III. As a result, each trajectory shape $q$ is viewed as an elements of the shape space, as shown in Fig. 4.

In order to analyze each step separately, we learn a step model for both left and right steps. From all the segmented training sequences, we first cluster segments to group together left steps and right steps. So as to handle possible noisy



Fig. 4. Spatio-temporal representation of steps. The skeleton sequence is regarded as a motion trajectory in a $3 J$-dimensional space whose shape is interpreted in the shape space.

segments, we employ the hierarchical clustering algorithm with the distance $d_{\mathcal{S}}$ to compare two shapes. From the resulted dendrogram, we select a threshold as stopping criterion resulting in a set of clusters. We then select the two larger clusters corresponding to left and right steps. The remaining samples are considered as outliers and discarded in the learning process. Then, for each cluster, we learn a statistical model by analyzing shape variations among all samples belonging to the same cluster. We assume that the distribution of shapes within a cluster follows a multivariate normal model. To handle the non-linearity of the shape space, a common way is to consider the tangent space at a reference point as a linear approximation of the neighborhood of the reference point. As sample shapes belong to a same cluster, we can assume that they lie in a small neighborhood. First, we compute the mean shape $\mu$ using the Riemannian center of mass of Eq. (3). Then, each sample shape $q_{i}$ is projected in the tangent space at the mean shape $T_{\mu} \mathcal{S}$ using the inverse exponential map operator [15] defined as:

$$
v_{i}=\exp _{\mu}^{-1}\left(q_{i}\right)=\frac{\theta}{\sin \theta}\left(q_{i}-\cos (\theta) \mu\right),
$$

where $\theta=d_{\mathcal{S}}\left(\mu, q_{i}\right)$. Such tangent vector $v_{i} \in \mathbb{R}^{3 J}$, called velocity vector, captures the shape difference between the mean $\mu$ and $q_{i}$. The original shape $q_{i}$ can be retrieved from the velocity vector $v_{i}$ by using the exponential map operator [15] defined as:

$$
q_{i}=\exp _{\mu}\left(v_{i}\right)=\cos \left(\left\|v_{i}\right\|\right) \mu+\sin \left(\left\|v_{i}\right\|\right) \frac{v_{i}}{\left\|v_{i}\right\|} .
$$

By locally analyzing a velocity vector $v_{i}(t)$ for each parameterized $t$, we obtain the local deformation between the point $\mu(t)$ of the mean shape $\mu$ to the corresponding point $q_{i}(t)$ of the shape $q_{i}$. We assume that the variations of velocity vectors around the mean are mostly restricted to a $m$-dimensional subspace called principal subspace. Using the velocity vectors $\left\{v_{i}\right\}$, we can apply Principal Component Analysis (PCA) to identify such principal subspace. Finally, tangent vectors $v_{i}$ are projected into the principal subspace, resulting in $\tilde{v}_{i}$, and the multivariate normal distribution is learned using the covariance matrix $\Sigma \in \mathbb{R}^{m \times m}$ computed from the set of $\tilde{v}_{i}$. Its corresponding probability density function is defined as:

$$
f(\tilde{v})=\frac{1}{(2 \pi)^{n / 2}|\Sigma|^{1 / 2}} e^{-\frac{1}{2} \tilde{v}^{T} \Sigma^{-1} \tilde{v}} .
$$

We learn such multivariate normal distribution for both clusters in order to identify a step model for both right and 
left steps. Fig. 5 illustrates the multivariate normal distribution on the tangent space at the mean shape $\mu$.

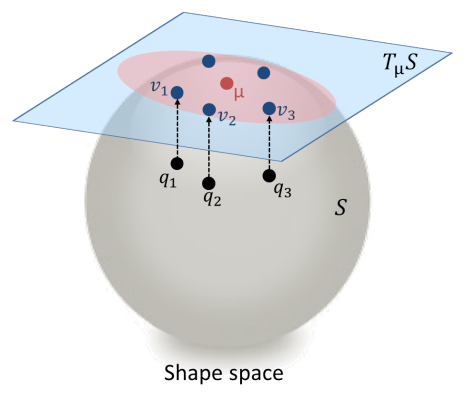

Fig. 5. Learning multivariate normal distribution on shape space. From the set of shapes $\left\{q_{i}\right\}$ (black), the mean shape $\mu$ (red) is computed using Eq. (3). Then, each shape is projected in the tangent space at $\mu$, resulting on the set $\left\{v_{i}\right\}$ (blue), which is finally used to learn the principal subspace and the multivariate normal distribution represented as an ellipsoid.

In the test step, we evaluate the gait sequences using the two learned step models. First, the test sequence is segmented using the method described in Sect. IV. Then, each resulting segment $s$ is evaluated using the learned models. To this end, the corresponding shape $q_{s}$ is projected into the principal subspace resulting in $\tilde{v}_{s}$. The $\log$-probability $L(s)$ that a segment $s$ has been generated by the multivariate distribution is thus defined as:

$$
L(s)=-\frac{1}{2} \ln (|\Sigma|)-\frac{1}{2} \tilde{v}_{s}^{T} \Sigma^{-1} \tilde{v}_{s}-\frac{n}{2} \ln (2 \pi) .
$$

Such log-probability is computed for both step models and the highest is kept. As a result, a test sequence is represented as a set of $\log$ probabilities $\{L(1), \ldots L(s), \ldots, L(S)\}$, being $S$ the number of segments in the test sequence. Such sequence is then evaluated differently according to the context as described in Sect. VI.

\section{EXPERIMENTAL RESULTS}

We first propose to validate our gait step learning model by observing gait sequences generated by our model. Then, we evaluate the effectiveness of our gait analysis method in the context of abnormal gait detection of people walking or climbing stairs. Finally, we explore the use of such method for the task of person re-identification through gait analysis.

\section{A. Gait sequences generation}

One advantage of learning statistical models is that they can be employed to generate random observations. We propose to successively use the two learned distributions corresponding to a right step and a left step to generate random gait sequences. For a given model, we first compute a random sample by associating random coefficients to the learned principal subspace. Let $\left\{\sigma_{k}, U_{k}\right\}$ be the $K$ eigen values and eigen directions resulted from a PCA in the tangent space, as explained in section $\mathrm{V}$. Then, a random tangent vector can be generated as: $\widehat{v}=\sum_{k=1}^{K} z_{k} \sigma_{k} U_{k}$, where $z_{k}$ are random values taken between -1 and 1 . The resulted tangent vector $\widehat{v}$ is then mapped in the shape space using the exponential map operator (eq. 5), resulting in a random shape $\widehat{q}$. Finally the corresponding skeleton sequence of a walking step is retrieved from $\widehat{q}$ using eq. 2. By successively combining a random left step and a random right step, we can generate a random gait sequence. Fig. 6 shows an example of two random sequences including one gait cycle starting by the right step and left step, respectively.

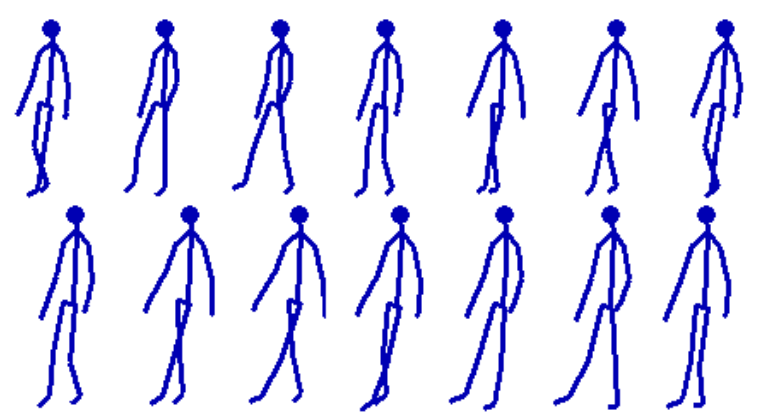

Fig. 6. Example of random gait sequences generated by the two statistical models corresponding to right and left steps.

\section{B. Abnormal gait detection}

For abnormal gait detection, the proposed approach is evaluated on the DAI gait [2] and SPHERE [18] datasets of people walking and climbing stairs, respectively.

1) DAI gait dataset: This dataset [2] has been recorded using Kinect 2. It includes sequences of seven subjects walking toward the camera. Each subject performed four times a normal walking. In addition, two gait anomalies are established, a knee injury that implies that the knee cannot be bent, and a second injury where one foot is dragged towards the other, which usually happens when a mobility aid such as a crutch is employed. These anomalies are performed for the right and left legs leading to four types of abnormal gait. Each subject performs once each type of anomaly. As a result, the dataset contains 56 gait sequences. In order to fairly compare our method with the work proposed in [2], we consider the same two scenarios: Scenario 1: The training set contains three out of four normal gait sequences of each subject and the testing set includes the remaining normal and abnormal sequences; Scenario 2: The training set contains normal sequences from four subjects and the testing set includes normal and abnormal sequences of the three remaining actors. This second scenario is more realistic as each subject is used only once in either training or test set. In each scenario, only normal sequences are used for training and the classification is performed by thresholding the similarity score between training sequences and test sequences. The similarity measure $D$ is defined as the mean log-probability over all $S$ segments of the test sequence: $D=\frac{1}{S} \sum_{s=1}^{S} L(s)$. Each sequence is classified as normal or abnormal according to this similarity measure and a threshold selected empirically, similarly to [2]. Finally, the precision and recall measures are computed according to correct classifications and the overall abnormal detection accuracy of the approach is evaluated using the $F_{1}$-score denoted as: $F_{1}=2 \times \frac{\text { precision } \times \text { recall }}{\text { precision }+ \text { recall }}$. 


\begin{tabular}{|l|c|c|}
\hline Method & Scenario 1 & Scenario 2 \\
\hline Chaaraoui et al. [2] & $\mathbf{0 . 9 8}$ & 0.85 \\
Our & $\mathbf{0 . 9 8}$ & $\mathbf{0 . 9 6}$ \\
\hline
\end{tabular}

TABLE I

ABNORMAL DETECTION RESULTS ON DAI GAIT DATASET IN COMPARISON WITH THE METHOD PROPOSED IN [2].

Results are reported in Table I. Compared to [2], we obtain same accuracy for the first scenario, and a significant improvement for the second scenario. This shows the effectiveness of our method for abnormal gait detection even if the test subject is unknown. In addition, our method allows analyzing the deformation of a test step with respect to a template. For instance, let $v_{s}$ be the velocity vector of a test shape trajectory $q_{s}$ projected in the tangent space of the corresponding learned step model. As we know the correspondence between each of the $3 J$ dimensions and the joints coordinates, we can compute the magnitude of each 3-tuple to quantify the deformation of each joint along the time. Fig. 7 illustrates such deformation of normal and abnormal steps with respect to a mean step. This analysis allows localizing which part of the body is more responsible of the gait deficiency. This could provide a feedback to the actor on where the movement should be improved.

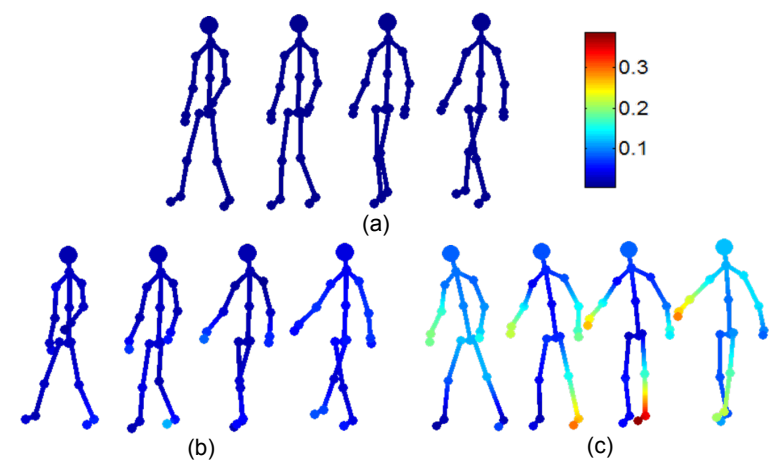

Fig. 7. Left step shape variation around the mean step (a) of a normal step (b) and abnormal step (c). Variation of abnormal step is higher than normal step and mainly localized in the left leg.

2) SPHERE dataset: We evaluate our approach in a different abnormal gait detection context. We use the SPHERE dataset [18], which includes sequences of 12 people climbing stairs with three types of abnormal gait: Left-leg Lead (LL)subjects walk up the stairs always using their left leg to move to the next upper step; Right-leg Lead (RL)—subjects walk up the stairs always using their right leg to move to the next upper step; Freeze of Gait (FoG) — subjects stop their movement once or twice while climbing stairs. Following the same protocol employed in [18], the first six subjects are used for training and the six remaining subjects for test. For this experiment the evaluation is done for each step separately. To evaluate each step $s$ of a sequence, we use the $\log$-probability $L(s)$ computed using Eq. (7). Similarly to [18], we use a threshold determined empirically to classify a step as normal or abnormal.
So as to evaluate the method, abnormal gait detection results are compared with a ground truth.

\begin{tabular}{|l|l|ccc|}
\hline \multicolumn{2}{|c|}{ Type of event } & LL & RL & FOG \\
\hline \multicolumn{2}{|c|}{ Number of occurrences } & 21 & 25 & 12 \\
\hline \multirow{2}{*}{ False Positives } & Paiement et al. [18] & $\mathbf{0}$ & $\mathbf{0}$ & 2 \\
& Our & $\mathbf{0}$ & $\mathbf{0}$ & $\mathbf{0}$ \\
\hline \multirow{2}{*}{ True Positives } & Paiement et al. [18] & 19 & 23 & $\mathbf{1 2}$ \\
& Our & $\mathbf{2 1}$ & $\mathbf{2 4}$ & 5 \\
\hline \multirow{2}{*}{ False Negatives } & Paiement et al. [18] & 2 & 2 & $\mathbf{0}$ \\
& Our & $\mathbf{0}$ & $\mathbf{1}$ & 7 \\
\hline
\end{tabular}

TABLE II

ABNORMAL GAIT DETECTION RESULTS ON SPHERE DATASET IN COMPARISON WITH PAIEMENT ET AL. [18].

Results are reported in Table II in comparison with [18]. We do not report results obtained by [2] as they employed a sequence-based classification. We can see that our method obtains better detection results for the LL and RL cases. Fig. 8 shows the log-probability obtained for each step of a normal sequence and a RL sequence. We can see that the log-likelihood of abnormal steps falls below the threshold. However, our method does not accurately detect the FOG abnormal gaits. This can be explained by the fact that stopping the motion does not significantly change the shape of the motion trajectory. Thus, our shape analysis method is not able to detect this type of abnormal gait.


Fig. 8. Log-probabilities of left (orange) and right (green) steps obtained for normal sequence (top) and RL sequence (bottom). We can see that the logprobability of deficient left step falls below the threshold (black).

\section{Person re-identification}

Finally, we propose to evaluate our method for the task of person re-identification. To this end, we employ the frontal gait dataset [5]. However, only a short version of this dataset (5 subjects) is publicly available and we were not able to obtain the full dataset of 29 subjects. As a result, we can not fairly compare our method with the work proposed in [5]. To evaluate the person re-identification capability of our method, we learn both step models for each subject separately. During classification, log-probabilities are computed for each subject and the 
mean log-probability is used as similarity measure. As a result, a test sequence has five similarity measures corresponding to the five subjects. The sequence is finally classified as the subject whose similarity measure is the highest. We employ a three-fold cross validation protocol to evaluate the approach. For each of the five subjects, three gait sequences are available. For each fold, we used two sequences for training and the remaining one for test. We obtain an average accuracy of $93.3 \%$ of accuracy among the three folds. It corresponds to only one misclassified sequence. Even if these experiments are carried out on a very small database, this result shows the potential of our method for person re-identification through gait analysis. In addition, we note that our method only analyzes the gait signature of subjects without any soft biometrics information, like subject height or stride length, as it is common in the state-of-the-art.

\section{CONCLUSIONS}

In this paper, we focus on gait analysis using skeleton data provided by depth sensors. We first identify each individual step of the gait sequence by analyzing the evolution of the legs motion. Then, we consider the shape variation of motion trajectories in a Riemannian shape space to learn statistical models for the left and right step. These models are used to both generate random gait sequences and evaluate the quality of test gait sequences. Experimental results demonstrate the effectiveness of the proposed approach in the context of abnormal gait detection. However, experiments also show that our method is not able to accurately detect 'static' abnormalities like the 'freeze of gait'. Such a limitation will be part of our future work. Moreover, promising results obtained for the task of gait recognition motivate us to consider this challenge on larger datasets as future work. In addition, we would like to investigate the use of such statistical models to estimate the motion of missing or noisy body part that may appear in a real-world context. Finally, we plan to extend the motion analysis to different type of movements in several contexts like rehabilitation or sport gestures optimization.

\section{ACKNOWLEDGMENT}

This work was partially supported by the FUI project MAGNUM 2 and the Programme dInvestissements dAvenir (PIA) and Agence Nationale pour la Recherche (grant ANR-11EQPX-0023), and European Founds for the Regional Development (Grant FEDER- Presage 41779).

\section{REFERENCES}

[1] I. Barros Barbosa, M. Cristani, A. Del Bue, L. Bazzani, and V. Murino Re-identification with RGB-D sensors. In Eu. Conf. on Computer Vision Workshop, pages 433-442, Zurich, Swiss, Sept. 2012.

[2] A. Chaaraoui, J. Padilla-Lpez, and F. Flrez-Revuelta. Abnormal gait detection with rgb-d devices using joint motion history features. In IEEE Int. Conf. and Workshops on Automatic Face and Gesture Recog., Ljubljana, Slovenia, May 2015.

[3] A. A. Chaaraoui, P. Climent-Perez, and F. Florez-Revuelta. A review on vision techniques applied to human behaviour analysis for ambientassisted living. Expert Systems with Applications, 39(12):10873-10888, 2012.
[4] P. Chattopadhyay, A. Roy, S. Sural, and J. Mukhopadhyay. Pose depth volume extraction from RGB-D streams for frontal gait recognition. Journal of Visual Communication and Image Representation, 25(1):5363, 2014.

[5] P. Chattopadhyay, S. Sural, and J. Mukhopadhyay. Frontal gait recognition from occluded scenes. Pattern Recognition Letters, 63(1):9-15, 2015.

[6] V. Cimolin and M. Galli. Summary measures for clinical gait analysis: A literature review. Gait \& Posture, 39(4):1005-1010, 2014.

[7] M. Devanne, A. Wannous, S. Berretti, P. Pala, M. Daoudi, and A. Del Bimbo. Combined shape analysis of human poses and motion units for action segmentation and recognition. In IEEE Int. Conf. and Workshops on Automatic Face and Gesture Recog., Ljubljana, Slovenia, May 2015.

[8] M. Devanne, H. Wannous, S. Berretti, P. Pala, M. Daoudi, and A. Del Bimbo. 3D human action recognition by shape analysis of motion trajectories on riemannian manifold. IEEE Trans. on Cybernetics, 45(7):1340-1352, 2014.

[9] H. Drira, B. Ben Amor, A. Srivastava, M. Daoudi, and R. Slama. 3D face recognition under expressions, occlusions, and pose variations. IEEE Trans. Pattern Analysis and Machine Intelligence, 35(9):2270 2283, 2013.

[10] M. Gabel, R. Gilad-Bachrach, E. Renshaw, and A. Schuster. Full body gait analysis with kinect. In Engineering in Medicine and Biology Society $(E M B C)$.

[11] F. Gomez-Caballero, T. Shinozaki, S. Furui, and K. Shinoda. Statistical person verification using behavioral patterns from complex human motion. In New Trends in Image Analysis and Processing ICIAP, Naples, Italy, Sept. 2013

[12] J. Hausdorff, G. Yogev, S. Springer, E. Simon, and N. Giladi. Walking is more like catching than tapping: gait in the elderly as a complex cognitive task. Experimental Brain Research, 164(4):541-548, 2005.

[13] S. H. Joshi, E. Klassen, A. Srivastava, and I. Jermyn. A novel representation for Riemannian analysis of elastic curves in $R^{n}$. In Proc IEEE Int. Conf. on Computer Vision and Pattern Recognition, pages 1-7, Minneapolis, MN, USA, June 2007.

[14] H. Karcher. Riemannian center of mass and mollifier smoothing. Comm. on Pure and Applied Math., 30:509-541, 1977.

[15] S. Kurtek, A. Srivastava, E. Klassen, and Z. Ding. Statistical modeling of curves using shapes and related features. Journal of the American Statistical Association, 107(499):1152-1165, 2012.

[16] B. Kwolek and M. Kepski. Human fall detection on embedded platform using depth maps and wireless accelerometer. Computer Methods and Programs in Biomedicine, 117(3):489-501, 2014.

[17] M. Meng, H. Drira, M. Daoudi, and J. Boonaert. Detection of abnorma gait from skeleton data. In the 11th International Joint Conference on Computer Vision, Imaging and Computer Graphics Theory and Applications, Porto, Portugal, 2016.

[18] A. Paiement, L. Tao, S. Hannuna, M. Camplani, D. Damen, and M. Mirmehdi. Online quality assessment of human movement from skeleton data. In Proc. of British Machine Vision Conf., pages 1-12, Nottingham, UK, Sept. 2014.

[19] O. Popoola and K. Wang. Video-based abnormal human behavior recognitiona review. IEEE Trans. on Systems, Man, and Cybernetics, Part C: Applications and Reviews, 42(6):865-878, 2012.

[20] J. Preis, M. Kessel, M. Werner, and C. Linnhoff-Popien. Gait recognition with kinect. In Proc. of the First Workshop on Kinect in Pervasive Computing, Ljubljana, Slovenia, May 2012.

[21] S. Sivapalan, D. Chen, S. Denman, S. Sridharan, and C. Fookes. Gait energy volumes and frontal gait recognition using depth images. In Int. Joint Conf. on Biometrics (IJCB), 2011.

[22] T. Wren, G. Gorton III, S. Ounpuu, and C. Tucker. Efficacy of clinical gait analysis: A systematic review. Gait \& Posture, 34(2):149-153, 2011.

[23] X. Xu, R. McGorry, L. Chou, J. Lin, and C. Chang. Accuracy of the microsoft kinect for measuring gait parameters during treadmill walking. Gait \& Posture, 42(2):145-151, 2015.

[24] M. Zanfir, M. Leordeanu, and C. Sminchisescu. The moving pose: An efficient $3 \mathrm{~d}$ kinematics descriptor for low-latency action recognition and detection. In Proc. IEEE Int. Conf. on Computer Vision (ICCV), pages 2752-2759, Sydney, Australia, Dec. 2013. IEEE.

[25] H. Zhang and W. Zhou. Fuzzy segmentation and recognition of continuous human activities. In Proc. IEEE Int. Conf. on Robotics and Automation, pages 6305-6312. IEEE, 2014. 